\title{
Reply to "Retained placenta accreta with marked vascularity, uterine artery pseudoaneurysm, and placental polyp: overlapping clinical entities?"
}

\author{
Akihiro Takeda ${ }^{1} \mathbb{D} \cdot$ Wataru Koike ${ }^{2}$ \\ Received: 28 October 2017 / Accepted: 10 November 2017 / Published online: 16 November 2017 \\ ○) Springer-Verlag GmbH Germany, part of Springer Nature 2017
}

\section{Dear Editor,}

We thank Dr. Matsubara and colleagues for their interest in our work [1] and their comments [2].

Proposed their concept, in which retained placenta accreta with marked vascularity, uterine artery pseudoaneurysm, and placental polyp could be overlapping clinical entities, could be potentially interesting to verify. However, due to our limited experiences in this issue, we apologize to say that this proposal appeared to be beyond our scope aimed in this current research [1].

We have already understood that uterine pathological condition induced by retained placenta accreta after abortion or delivery could be variable. Its manifestation usually appeared to be different case by case, potentially depending on the degree of degeneration of retained villous tissue and induced vascular network surrounding the invasive placenta.

However, under clinical setting, it may be sometimes difficult to observe the disease process such as progression without intervention when acute condition such as significant hemorrhage occurs. Therefore, we assume that co-existence and transition of proposed three morbid conditions could be observed only in limited occasions, even though they exist.

About Fig. 2d, they commented that this angiographic image shows similarity to the image observed in pseudoaneurysm [2]. However, we think that this structure with hypervascular stain shows the bundle of arterio-venous shunt-like structure (this becomes clear if you simply

Akihiro Takeda

gyendoscopy@gmail.com

1 Department of Obstetrics and Gynecology, Gifu Prefectural Tajimi Hospital, 5-161 Maebata-cho, Tajimi, Gifu 507-8522, Japan

2 Department of Radiology, Gifu Prefectural Tajimi Hospital, 5-161 Maebata-cho, Tajimi, Gifu 507-8522, Japan magnify the image) similarly as shown in three-dimensional computerized tomographic angiography (Fig. 2c), rather than classically defined sac-like structure observed in uterine artery pseudoaneurysm [3].

Retained placenta accreta is an old issue; however, as Dr. Matsubara commented, its pathophysiological characterization should continue to be investigated, by introducing newer diagnostic and therapeutic modalities for life-saving as well as fertility perseveration of women.

We truly appreciate the readers for commenting on this important issue and hope to have an opportunity to discuss further after providing their expertise with scientific data based on sufficient evidence on this important issue.

Author contribution AT and WK: identification of the significance. AT: manuscript writing.

\section{Compliance with ethical standards}

Conflict of interest The authors report no conflict of interest.

Funding No funding was provided for this research.

Human participants or animal experiments This article does not contain any studies with human participants or animals.

\section{References}

1. Takeda A, Koike W (2017) Conservative endovascular management of retained placenta accreta with marked vascularity after abortion or delivery. Arch Gynecol Obstet 296:1189-1198

2. Matsubara S, Takahashi, Suzuki T. Retained placenta accreta with marked vascularity, uterine artery pseudoaneurysm, and placental polyp: overlapping clinical entities? Arch Gynecol Obstet

3. Takeda A, Koike W, Imoto S, Nakamura H (2014) Conservative management of uterine artery pseudoaneurysm after laparoscopicassisted myomectomy and subsequent pregnancy outcome: case series and review of the literature. Eur J Obstet Gynecol Reprod Biol 182:146-153 